\title{
UNIQUENESS THEOREMS FOR HARMONIC FUNCTIONS OF EXPONENTIAL GROWTH
}

\author{
DORON ZEILBERGER
}

\begin{abstract}
Two uniqueness theorems for harmonic functions of exponential growth are proved. The first is a generalization to $R^{n}$ of a theorem proved by Boas [1] for $R^{2}$.
\end{abstract}

1. Introduction and statement of results. The purpose of this paper is to prove the following two theorems.

THEOREM A. Let $u$ be a real-valued harmonic function in $R^{n}$ satisfying $|u(x)|<C e^{A|x|}$, where $A<\pi,|x|=\sum_{i=1}^{n}\left|x_{i}\right|$ and $C$ is a constant. If $u$ vanishes on the integer lattice points of the hyperplanes

$$
x_{n}=0, \quad \text { and } x_{n}=a \quad\left(|a| \leqslant(1 /(n-1))^{1 / 2}\right),
$$

then it vanishes identically.

THEOREM B. Let $u$ be as above and suppose both $u$ and $\partial u / \partial x_{n}$ vanish on the integer lattice points of $x_{n}=0$; then $u$ vanishes identically.

Theorem A is a generalization of a theorem of Boas [1], who proved it for $n=2$. Boas used the fact that in the two-dimensional case every real-valued entire harmonic function is the real part of an entire (analytic) function. Evidently, this method does not generalize to higher dimensions. Our strategy will be, instead, to view $u$ as a "distribution" (i.e., a continuous linear functional) on the test space of bounded analytic functions on the polystrip $\times_{i=1}^{n}\left\{\left|\operatorname{Im} t_{i}\right|<A^{\prime \prime}\right\} \subset \mathbf{C}^{n}$ for $A^{\prime \prime}>A$. The referee has kindly informed us that Rao [2] has proved Theorem A by different methods.

2. Proof of the results. We shall proceed by a sequence of lemmas.

Lemma 1. If $u$ is harmonic in $R^{n}$ and $|u(x)|<C e^{A|x|}$, then any partial derivative of $u$ enjoys the same properties.

Proof. For any $x_{0} \in R^{n}$ look at the Poisson representation formula for the ball $\left\|y-x_{0}\right\|<1$, differentiate under the integral sign and estimate.

Let $\mathscr{Q}$ be the class of analytic functions of $n$ complex variables of the form

Received by the editors February 3, 1976.

AMS (MOS) subject classifications (1970). Primary 31B05; Secondary 31B10, 30A98.

Key words and phrases. Harmonic functions, Banach spaces of holomorphic functions, Green's formula. 


$$
\hat{v}(t)=(2 \pi)^{n / 2} \int v(x) e^{i x t} \quad\left(x t=\sum x_{i} t_{i}\right),
$$

where $v \in C_{0}^{\infty}\left(R^{n}\right)$. All these functions are bounded in

$$
K_{A^{\prime \prime}}={\underset{i=1}{\times}}_{i=1}^{n}\left\{\left|\operatorname{Im} t_{i}\right|<A^{\prime \prime}\right\} \quad \text { for every } A^{\prime \prime} .
$$

Define a linear functional on $\mathbb{Q}$ by

$$
T_{u}\left((2 \pi)^{n / 2} \int_{R^{n}} v(x) e^{i x t}\right)=\int_{R^{n}} u(x) v(x) .
$$

The next lemma will show that $T_{u}$ can be extended continuousiy to $\mathcal{H}_{A^{\prime \prime}}$, the Banach space of bounded holomorphic functions on $K_{A^{\prime \prime}}$, provided $A^{\prime \prime}>A$.

Lemma 2. Let $u$ be harmonic in $R^{n}$ and satisfy $|u(x)|<C e^{A|x|}$. Let $A^{\prime \prime}>A$; then $T_{u}$ defined on $\Theta$ by (2.1) can be extended to be a continuous linear functional on the Banach space $\mathcal{H}_{A^{\prime \prime}}$, which consists of bounded analytic functions on $K_{A^{\prime \prime}}$ where the norm is given by $\|f\|_{A^{\prime \prime}}=\sup _{t \in K_{A}}|f(t)|$.

Proof. Let $A<A^{\prime}<A^{\prime \prime}$ and let $R_{+}=[0, \infty), R_{-}=(-\infty, 0]$. Then

$$
U_{ \pm \pm \cdots \pm}(t)=(2 \pi)^{-n / 2} \int_{R_{ \pm} \times R_{ \pm} \times \cdots \times R_{ \pm}} u(x) e^{-i x t} d x
$$

belongs to $L^{2}\left(X_{i=1}^{n}\left\{\operatorname{Im} t_{i}=\mp A^{\prime}\right\}\right)$, and for $\hat{v} \in \mathbb{Q}$,

$$
\begin{aligned}
T_{u}(\hat{v}) & =\sum_{ \pm} \int_{R_{ \pm} \times \cdots \times R_{ \pm}} u(x) v(x) \\
& =\sum_{ \pm} \int_{X_{i=1}^{n}\left\{\operatorname{Im} t_{i}=\mp A^{\prime}\right\}} U_{ \pm \cdots \pm}(t) \hat{v}(t) d t_{1} \cdots d t_{n} .
\end{aligned}
$$

The sums in (2.2) each contain $2^{n}$ terms, corresponding to all possible choices of sign. Let us consider the term in the sum on the right-hand side of (2.2) involving $U_{-} \ldots_{-}(t)$, and let us write, for the moment, $\Omega=R_{-} \times R_{-}$ $\times \cdots \times R_{-}$. Then, by Green's formula,

$$
\begin{aligned}
(2 \pi)^{n / 2} U_{-} & -\cdots-(t)=\int_{\Omega} u(x) e^{-i x t} d x=\int_{\Omega} u(x) \Delta\left(\frac{-e^{-i x t}}{t_{1}^{2}+\cdots+t_{n}^{2}}\right) \\
= & -\int \Delta u(x) \frac{e^{-i x t}}{t_{1}^{2}+\cdots+t_{n}^{2}}+\int_{\partial \Omega} u(x) \frac{\partial}{\partial n}\left[\frac{-e^{-i x t}}{t_{1}^{2}+\cdots+t_{n}^{2}}\right] d \sigma \\
& -\int_{\partial \Omega} \frac{\partial u}{\partial n} \frac{-e^{-i x t}}{t_{1}^{2}+\cdots+t_{n}^{2}} d \sigma .
\end{aligned}
$$

The first term on the right-hand side of (2.3) vanishes since $u$ is harmonic. Now $\partial \Omega$ consists of $n$ pieces: $\partial \Omega=\cup_{i=1}^{n}\left\{x_{i}=0\right\} \cap \Omega$.

Let us consider the contribution from the face $x_{1}=0$. Here $\partial / \partial n=\partial / \partial x_{1}$ and 


$$
\begin{gathered}
\int_{\left\{x_{1}=0\right\} \cap \Omega} u(x) \frac{\partial}{\partial n}\left(\frac{-e^{-i x t}}{t_{1}^{2}+\cdots+t_{n}^{2}}\right) d x_{2} \cdots d x_{n} \\
=\int_{\left\{x_{1}=0\right\} \cap \Omega} u\left(0, x_{2}, \ldots, x_{n}\right) \frac{i t_{1} e^{-i x t} d \sigma}{t_{1}^{2}+\cdots+t_{n}^{2}}
\end{gathered}
$$

and

$$
\begin{aligned}
\int_{\left\{x_{1}=0\right\} \cap \Omega} \frac{\partial u}{\partial n}(x) \frac{e^{-i x t} d \sigma}{t_{1}^{2}+\cdots+t_{n}^{2}} \\
=\int_{\left\{x_{1}=0\right\} \cap \Omega} \frac{\partial u}{\partial x_{1}}\left(0, x_{2}, \ldots, x_{n}\right) \\
\quad-\frac{i t_{1} \exp \left(-i x_{2} t_{2} \cdots \cdots-i x_{n} t_{n}\right)}{t_{1}^{2}+\cdots+t_{n}^{2}} d x_{2} \cdots d x_{n}
\end{aligned}
$$

Now look at (2.2), the contribution from (2.4) is

$$
\begin{aligned}
& \int_{\left\{\operatorname{Im} t_{i}=A^{\prime}\right\} \times \times_{2}^{n}\left\{\operatorname{Im} t_{i}=A^{\prime}\right\}} \hat{v}(t) d t_{1} \cdots d t_{n} \\
& \quad \times \int_{\left\{x_{1}=0\right\} \cap \Omega} u\left(0, x_{2}, \ldots, x_{n}\right) \frac{i t_{1} \exp \left(-i x_{2} t_{2}-\cdots-i x_{n} t_{n}\right)}{t_{1}^{2}+\cdots+t_{n}^{2}} d x_{2} \cdots \cdot d x_{n} .
\end{aligned}
$$

But there is a similar contribution, with an opposite sign, from integration on $\left\{\operatorname{Im} t_{1}=-A^{\prime}\right\} \times \times_{2}^{n}\left\{\operatorname{Im} t_{i}=A^{\prime}\right\}$. Let $\Gamma_{A^{\prime}}$ be the rectangular contour in the $t_{1}$-plane with sides $\pm i A^{\prime} \pm R$; then, as $R \rightarrow \infty$, the sum of these contributions is

$$
\int_{X_{2}^{n}\left\{\operatorname{Im} t_{i}=A^{\prime}\right\}} \phi\left(t_{2}, \ldots, t_{n}\right) d t_{2} \cdots d t_{n} \int_{\Gamma_{A^{\prime}}} \frac{\hat{v}\left(t_{1}, \ldots, t_{n}\right) i t_{1}}{t_{1}^{2}+\cdots+t_{n}^{2}} d t_{1}
$$

where

$$
\begin{aligned}
\phi\left(t_{2}, \ldots, t_{n}\right)= & \int_{\left\{x_{1}=0\right\} \cap \Omega} u\left(0, x_{2}, \ldots, x_{n}\right) \\
& \times \exp \left(-i x_{2} t_{2}-\cdots-i x_{n} t_{n}\right) d x_{2} \cdots d x_{n} .
\end{aligned}
$$

For fixed $t_{2}, \ldots, t_{n}$,

$$
\begin{aligned}
& \int_{\Gamma_{A^{\prime}}} \frac{\hat{v}\left(t_{1}, \ldots, t_{n}\right) i t_{1}}{t_{1}^{2}+\cdots+t_{n}^{2}} d t_{1} \\
& \quad=\left\{\begin{array}{ll}
\pi i\left\{\hat{v}\left(\tau_{1}, t_{2}, \ldots, t_{n}\right)+\hat{v}\left(-\tau_{1}, t_{2}, \ldots, t_{n}\right)\right\} \\
0 & \text { if }\left|\operatorname{Im} \tau_{1}\right|>A^{\prime},
\end{array} \text { if }\left|\operatorname{Im} \tau_{1}\right|<A^{\prime},\right.
\end{aligned}
$$

where $\tau_{1}=\tau_{1}\left(t_{2}, \ldots, t_{n}\right)$ is given by

$$
\tau_{1}^{2}+t_{2}^{2}+\cdots+t_{n}^{2}=0
$$

i.e., $\tau_{1}=i\left(t_{2}^{2}+\cdots+t_{n}^{2}\right)^{1 / 2}$. Now

$$
M_{A^{\prime}}=\left\{\left(t_{2}, \ldots, t_{n}\right) \in \mathbf{C}^{n-1}, \operatorname{Im} t_{2}=A^{\prime}, \ldots, \operatorname{Im} t_{n}=A^{\prime},\left|\operatorname{Im} \tau_{1}\right|<A^{\prime}\right\}
$$


is seen to be a compact subset of $\times_{i=2}^{n}\left\{\operatorname{Im} t_{i}=+A^{\prime}\right\}$, and we get that the contribution from the pair of boundary terms (obtained in (2.3)) considered is

$$
\begin{aligned}
\pi i \int_{M_{A^{\prime}}} \phi\left(t_{2}, \ldots, t_{n}\right)[\hat{v} & \left(\tau_{1}, t_{2}, \ldots, t_{n}\right) \\
& \left.+\hat{v}\left(-\tau_{1}, t_{2}, \ldots, t_{n}\right)\right] d t_{2} \cdots d t_{n},
\end{aligned}
$$

and its absolute value is $\leqslant$ constant $\|\hat{v}\|_{A^{\prime \prime}}$.

Similarly, if

$$
\begin{aligned}
\phi^{\prime}\left(t_{2}, \ldots, t_{n}\right)= & -\int_{\left\{x_{1}=0\right\} \cap R_{-} \times \cdots \times R_{-}} \frac{\partial u}{\partial x_{1}}\left(0, x_{2}, \ldots, x_{n}\right) \\
& \times \exp \left(-i x_{2} t_{2}-\cdots-i x_{n} t_{n}\right) d x_{2} \cdots d x_{n},
\end{aligned}
$$

the net contribution from the two terms in (2.2) involving $\phi^{\prime}\left(t_{2}, \ldots, t_{n}\right)$ is

$$
\int_{\left\{\operatorname{Im} t_{2}=A^{\prime}\right\} \times \cdots \times\left\{\operatorname{Im} t_{n}=A^{\prime}\right\}} \phi^{\prime}\left(t_{2}, \ldots, t_{n}\right) \int_{\Gamma_{A^{\prime}}} \frac{\hat{v}\left(t_{1}, \ldots, t_{n}\right)}{t_{1}^{2}+\cdots+t_{n}^{2}} d t_{1}
$$

which is equal to

$$
\begin{aligned}
\pi \int_{M_{A^{\prime}}} & \phi^{\prime}\left(t_{2}, \ldots, t_{n}\right) \\
& \cdot \frac{1}{\tau_{1}}\left[\hat{v}\left(\tau_{1}, t_{2}, \ldots, t_{n}\right)-\hat{v}\left(-\tau_{1}, t_{2}, \ldots, t_{n}\right)\right] d t_{2} \cdots d t_{n}
\end{aligned}
$$

which, in absolute value, is $\leqslant$ constant $\|v\|_{A^{\prime \prime}}$.

In a similar way we can consider all other terms of (2.2) and write it as a sum of $n 2^{n-1}$ terms of the form (2.6') and $n 2^{n-1}$ terms of the form (2.7'). The resulting formula defines $T_{u}(f)$ for every $f \in \mathcal{H}_{A^{\prime \prime}}$ and $T_{u}$ is a bounded linear functional on $\mathcal{H}_{A^{\prime \prime}}$.

Lemma. 3. For every $x \in R^{n}, T_{u}\left(e^{i x t}\right)=(2 \pi)^{-n / 2} u(x)$.

Proof. Let $K_{\varepsilon}$ be a $C^{\infty}$ compact support approximate identity; then $\int K_{\varepsilon}(y-x) e^{i y t} d t \rightarrow e^{i x t}$ in the topology of $\mathcal{H}_{A^{\prime \prime}}$ and

$$
\begin{aligned}
T_{u}\left(e^{i x t}\right) & =\lim _{\varepsilon \rightarrow 0} T_{u}\left(\int K_{\varepsilon}(y-x) e^{i y t} d y\right) \\
& =\lim _{\varepsilon \rightarrow 0}(2 \pi)^{-n / 2} \int K_{\varepsilon}(y-x) u(y)=(2 \pi)^{-n / 2} u(x) .
\end{aligned}
$$

LEMMA 4. There exist measures $d \mu_{1}, d \mu_{2}$ on $\left\{t_{n}=0\right\}=\mathbf{C}^{n-1}$ supported in the compact set

$$
\begin{array}{r}
L_{A^{\prime \prime}}=\left\{\left(t_{1}, \ldots, t_{n-1}\right) ;\left|\operatorname{Im} t_{1}\right|<A^{\prime \prime}, \ldots,\left|\operatorname{Im} t_{n-1}\right|<A^{\prime \prime},\right. \\
\left.\left|\operatorname{Re}\left(t_{1}^{2}+\cdots+t_{n-1}^{2}\right)^{1 / 2}\right|<A^{\prime \prime}\right\} .
\end{array}
$$

such that for every $f \in \mathcal{K}_{A^{\prime \prime}}$, 


$$
\begin{aligned}
T_{u}(f)= & \int f\left(t_{1}, \ldots, t_{n-1}, i\left(t_{1}^{2}+\cdots+t_{n-1}^{2}\right)^{1 / 2}\right) d \mu_{1} \\
& +\int f\left(t_{1}, \ldots, t_{n-1},-i\left(t_{1}^{2}+\cdots+t_{n-1}^{2}\right)^{1 / 2}\right) d \mu_{2} .
\end{aligned}
$$

In particular,

$$
\begin{aligned}
u(x)= & \int \exp \left(i x_{1} t_{1}+\cdots+i x_{n-1} t_{n-1}\right) \exp \left(-\left(t_{1}^{2}+\cdots+t_{n-1}^{2}\right)^{1 / 2} x_{n}\right) d \mu_{1} \\
& +\int \exp \left(i x_{1} t_{1}+\cdots+i x_{n-1} t_{n-1}\right) \exp \left(\left(t_{1}^{2}+\cdots+t_{n-1}^{2}\right)^{1 / 2} x_{n}\right) d \mu_{2} .
\end{aligned}
$$

Proof. Let $V_{A^{\prime \prime}}=\left\{\left(t_{1}, \ldots, t_{n}\right) \in K_{A^{\prime \prime}} ; t_{1}^{2}+t_{2}^{2}+\cdots+t_{n}^{2}=0\right\}$. Then by the proof of Lemma 2 , by adding all the terms like $\left(2.6^{\prime}\right)$ and $\left(2.7^{\prime}\right)$, we get that there exists a measure $d \nu$, supported in $V_{A^{\prime \prime}}$ such that for every $f \in \mathcal{H}_{A^{\prime \prime}}$, $T_{u}(f)=\int f d \nu$.

Let $d \nu=d \nu_{1}+d \nu_{2}$, where $d \nu_{1}$ is supported in

$$
\left\{\left(t_{1}, t_{2}, \ldots, i\left(t_{1}^{2}+\cdots+t_{n-1}^{2}\right)^{1 / 2}\right)\right\}
$$

and $d \nu_{2}$ is supported in

$$
\left\{\left(t_{1}, t_{2}, \ldots, t_{n-1},-i\left(t_{1}^{2}+\cdots+t_{n-1}^{2}\right)^{1 / 2}\right)\right\} .
$$

Let $d \mu_{1}, d \mu_{2}$ be the projections of $d \nu_{1}, d \nu_{2}$, respectively, on $t_{n}=0$. Then the lemma follows since $d \mu_{1}, d \mu_{2}$ are supported in the projection of $V_{A^{\prime \prime}}$ on $t_{n}=0$ which is $L_{A^{\prime \prime}}$.

Now we are in a position to prove the theorems.

Proof of Theorem A. Since $A<\pi$ we can choose $A<A^{\prime \prime}<\pi$. It is easily seen that $L_{A^{\prime \prime}}$ is contained in $\times_{i=1}^{n-1}\left\{\left|\operatorname{Im} t_{i}\right|<A^{\prime \prime}\right\} \times\left\{\left|\operatorname{Re} t_{i}\right|<A^{\prime \prime}\right\}$, and since $A^{\prime \prime}<\pi$, the span of $\left\{e^{i x t} ; x \in Z^{n-1}\right\}$, where $Z^{n-1}$ are the integer lattice points of $R^{n-1}$, is dense in the space of bounded holomorphic functions on $L_{A^{\prime \prime}}$. By (2.9), $d \mu_{1}+d \mu_{2} \equiv 0$ and

$$
\exp \left(-a\left(t_{1}^{2}+\cdots+t_{n-1}^{2}\right)^{1 / 2}\right) d \mu_{1}+\exp \left(a\left(t_{1}^{2}+\cdots+t_{n-1}^{2}\right)^{1 / 2}\right) d \mu_{2} \equiv 0 .
$$

Since $a \leqslant(1 /(n-1))^{1 / 2}$ it follows that $d \mu_{1}, d \mu_{2}$ are supported in $\left\{t_{1}^{2}\right.$ $\left.+\cdots+t_{n-1}^{2}=0\right\}$ and by (2.9), $u(x)$ is identically zero.

Proof of Theorem B. Applying $\partial / \partial x_{n}$ to (2.9) we get

$$
\begin{aligned}
\frac{\partial u}{\partial x_{n}}\left(x_{1}, \ldots, x_{n-1}, 0\right) & \\
= & -\int \exp \left(i x_{1} t_{1}+\cdots+i x_{n-1} t_{n-1}\right)\left(t_{1}^{2}+\cdots+t_{n-1}\right)^{1 / 2} d \mu_{1} \\
& +\int \exp \left(i x_{1} t_{1}+\cdots+i x_{n-1} t_{n-1}\right)\left(t_{1}^{2}+\cdots+t_{n-1}^{2}\right)^{1 / 2} d \mu_{2} .
\end{aligned}
$$

As in the proof of the Theorem A we get that

$$
d \mu_{1}+d \mu_{2} \equiv 0, \quad\left(t_{1}^{2}+\cdots+t_{n-1}^{2}\right)^{1 / 2}\left(d \mu_{1}-d \mu_{2}\right) \equiv 0 .
$$

Thus $d \mu_{1}=-d \mu_{2}$ is supported at the set $\left\{t_{1}^{2}+\cdots+t_{n-1}^{2}=0\right\}$, and by 
using (2.9) it once again follows that $u$ vanishes identically.

Acknowledgement. The author wishes to thank Professor Harry Dym, Dr. Amy Novick, and Dr. Akiva Gabor for helpful comments and discussions.

\section{REFERENCES}

1. R. P. Boas, Jr., A uniqueness theorem for harmonic functions, J. Approximation Theory 5 (1972), 425-427. MR 49 \#610.

2. N. V. Rao, Carlson theorem for harmonic functions in $R^{n}$ (to appear).

Department of Mathematics, Weizmann Institute of Science, Rehovot, IsRael 